IPM/P-2008/061

\title{
Non-relativistic CFT and Semi-classical Strings
}

\author{
Amin Akhavan ${ }^{a}$, Mohsen Alishahiha ${ }^{b}$, Ali Davody $^{a, b}$ and Ali Vahedi ${ }^{a, b}$ \\ a Department of Physics, Sharif University of Technology \\ P.O. Box 11365-9161, Tehran, Iran \\ ${ }^{b}$ School of physics, Institute for Research in Fundamental Sciences (IPM) \\ P.O. Box 19395-5531, Tehran, Iran \\ amin_akhavan@mehr.sharif.ir, alishah ,davody, vahedi @ipm.ir
}

\begin{abstract}
We study different features of 3D non-relativistic CFT using gravity description. As the corresponding gravity solution can be embedded into the type IIB string theory, we study semi-classical closed/open strings in this background. In particular we consider folded rotating and circular pulsating closed strings where we find the anomalous dimension of the dual operators as a function of their quantum numbers. We also consider moving open strings in this background which can be used to compute the drag force. In particular we find that for slowly moving particles, the energy is lost exponentially and the characteristic time is given in terms of the temperature, while for fast moving particles the energy loss goes as inverse of the time and the characteristic time is independent of the temperature.
\end{abstract}

Dedicated to Reza Mansouri on the occasion of his 60th birthday 


\section{Introduction}

AdS/CFT correspondence [1] has given us a useful tool to find weakly coupled descriptions for strongly coupled conformal field theories. The weakly coupled description generically is given in terms of a supergravity on a background containing an AdS part. Actually there is a one to one correspondence between objects in gravity side and those in the conformal field theory dual. In particular it is known that the symmetries of the conformal field theory can be geometrically realized in the gravity side as the isometries of the metric. For example the conformal group of the $d$-dimensional space-time, $S O(d, 2)$, is realized as the isometry of the $A d S_{d+1}$ geometry where the gravity is defined.

Encourage with the great success in describing relativistic strongly coupled conformal field theories, it is natural to look for a weakly coupled gravity descriptions for non-relativistic conformal field theories. Indeed there are several models, for example, in condensed mater where the theories are invariant under Schrödinger group which is essentially the conformal group of the non-relativistic field theories (for example see [2]). Therefore it is important to find the corresponding gravity dual.

Actually taking into account that the Schrödinger group, $S \operatorname{ch}(d-1)$, is a subgroup of the relativistic conformal group $S O(d, 2)$ and indeed can be obtained from it by taking non-relativistic limit (contraction), one would expect that the same procedure can be applied in the gravity side as well. Namely one expects that the geometry we are interested in could be given by a deformation of AdS geometry. In fact the corresponding gravity whose isometry is $S c h(d-1)$ has been proposed in $[3,4] 1]$ which is

$$
d s^{2}=\frac{r^{2}}{R^{2}}\left(2 d t d y+d x_{i}^{2}-\mu^{2} r^{2} d t^{2}\right)+\frac{R^{2}}{r^{2}} d r^{2},
$$

where $i=1,2, \cdots, d-1$. Here $\mu$ is a parameter which controls the deviation from AdS geometry. In other words $\mu$ is a parameter which characterizes the nonrelativistic nature of the dual field theory. More precisely, as we will see, the corresponding physical dimensionless parameter indeed $\mu R$. The above metric is invariant under the following rescaling of the coordinates

$$
t \rightarrow \lambda^{2} t, \quad x_{i} \rightarrow \lambda x_{i}, \quad y \rightarrow y, \quad r \rightarrow \lambda^{-1} r .
$$

This $d+2$-dimensional gravity is proposed to describe a $d$-dimensional non-relativistic CFT which is invariant under the following scaling

$$
t \rightarrow \lambda^{2} t, \quad x_{i} \rightarrow \lambda x_{i}, \quad \text { for } \quad i=1, \cdots, d-1 .
$$

Since our main knowledge of AdS/CFT duality has come from string theory, it is natural to pose the question whether this solution can be embedded in the ten

\footnotetext{
${ }^{1}$ For recent studies on AdS/non-relativistic field theories see [5-20] (See also [21]).
} 
dimensional superstring theories. If it does, then one may use our experiences in string theory to study some features of the non-relativistic CFTs.

The aim of this article is to study some features of (1+2)-dimensional nonrelativistic CFT using supergravity solution in type IIB string theory. Since we are dealing with string theory, it is natural to consider a semi-classical string in this background. Having this in our mind, we will first consider a folded rotating closed string [22] (for more details see, e.g. [23]) and evaluate the relation between its energy, $E$, and spin $S$. Using AdS/CFT dictionary this corresponds to an operator with anomalous dimension $\Delta=E$ and spin $S$. We find that, for $\mu R \ll 1$ the anomalous dimension gets logarithmic corrections similar to AdS case, though the coefficients are functions of $\mu R$, at least up to order we are considering. This might be interpreted as the fact that in going from relativistic to non-relativistic theory the anomalous dimension of the corresponding operators gets corrections. We will also study circular pulsating strings following Ref. [24]. In this case, in comparison with the AdS case, we find new behavior at subleading order which we would like to associate with the non-relativistic properties of the dual theory.

It is also interesting to study semi-classical open strings in this background. In the context of AdS/CFT correspondence an open string can be associated to a Wilson loop in the dual field theory, which in turn can be used to compute the effective potential between external objects (e.g. quark-anti quark potential). In our case at zero temperature we find the potential behaves as $l^{-2}$ as expected for non-relativistic CFT with dynamical scale $z=2$.

To explore non-relativistic properties of the dual field theory we will also consider a moving open string on the relevant geometry at finite temperature which may be thought of as a moving external object on the hot plasma 2 . In this case, unlike the relativistic case, we observe that there is no upper bound on the velocity of the moving object, as expected for a non-relativistic theory. We will also see that when the external object moves through the plasma very slowly it loses its energy exponentially as a function of time, while when it moves very fast the decay rate behaves as inverse of time.

We will also redo the drag force-like computations for the non-relativistic CFT at zero temperature. Being at zero temperature the moving objects still lose their energy, though unlike the finite temperature case, the characteristic time is given in terms of $\mu$.

The paper is organized as follows. In the next section we review the embedding of the relevant geometry in type IIB string theory where we will also present the general features of the dual non-relativistic CFT. In section three we will study semi-classical closed strings on the background presented in section two. In section four we consider moving open strings on the geometry generated by a black hole in the supergravity solution of section two. In the section five we study the drag force in the non-relativistic field theory at zero temperature. The last section is devoted

\footnotetext{
${ }^{2}$ When the dual theory is gauge theory the external object could be quark and the plasma can be made out of quark-gluon plasma.
} 
to discussions and conclusions.

\section{Supergravity description}

The supergravity solution we are interested in can be obtained from the $A d S_{5} \times S^{5}$ solution of type IIB supergravity solution via TsT transformation or by making use of the Null Melvin Twist procedure [25], [26-30]3

$$
d s^{2}=\left(\frac{r}{R}\right)^{2}\left[-\mu^{2} r^{2} d t^{2}+2 d t d \xi+d x_{i}^{2}\right]+\left(\frac{R}{r}\right)^{2}\left[d r^{2}+r^{2} d \mathcal{M}_{5}^{2}\right],
$$

where $d \mathcal{M}_{5}^{2}$ is the metric of the five dimensional internal space whose spin structure fixes the number of supersymmetries preserved by the background. For example the internal space could be a five sphere. Indeed this solution has been obtained in [26] in the context of light like dipole field theory where it was shown that the solution preserves eight supercharges.

To proceed let us consider the dilaton field, $\phi$ which can be treated as a massless scalar field in the bulk supergravity. In case of $A d S_{5} \times S^{5}$, the dilaton field is dual to the operator $\mathcal{O}=\operatorname{Tr} F^{2}$ with $\Delta=4$ whose two point function is

$$
\langle\mathcal{O}(x, t) \mathcal{O}(0)\rangle \sim \frac{1}{|X|^{8}}, \quad \text { with }|X|=\sqrt{-t^{2}+x^{2}}
$$

In our case we would like to study the dilaton field in the background (2.1). Setting $\phi=e^{-i M \xi} e^{-i \omega t+i k \cdot x} \psi(r)$ the equation of motion for the dilaton becomes

$$
\frac{1}{r^{3}} \partial_{r}\left(r^{5} \partial_{r} \psi\right)-\left(\mu^{2} M^{2} R^{4}+\frac{q^{2} R^{4}}{r^{2}}\right) \psi=0,
$$

where $q^{2}=2 M \omega+k^{2}$. Using AdS/CFT procedure one can compute the two point function of the dual operator in the non-relativistic field theory. This has essentially been done in $[3,4]$. The result is

$$
\langle\mathcal{O}(x, t) \mathcal{O}(0)\rangle \sim t^{-\Delta} e^{-\frac{i M x^{2}}{2 t}}=\frac{\left(x^{2} / t\right)^{\Delta} e^{-\frac{i M x^{2}}{2 t}}}{x^{2 \Delta}},
$$

where $\Delta=2+\sqrt{4+\mu^{2} M^{2} R^{4}}$ is the dimension of the dual operator.

As we have already anticipated in the above computations, by the scaling arguments, the solution of (2.3) depends only on $q^{2} R^{4} / r^{2}$. Therefore the UV/IR relation in the bulk and boundary theories should be as follows [31]

$$
\delta t \sim \frac{M R^{4}}{r^{2}}, \quad \delta x \sim \frac{R^{2}}{r} .
$$

\footnotetext{
${ }^{3}$ Note that the whole solution beside the metric contains a non-zero RR 4 -form as well as non-zero NSNS B field whose explicit forms are not important for what follows. The dilaton is constant as expected.
} 
Actually for the relativistic case where both $x$ and $t$ scale the same, the UV/IR relations are the same, i.e. $\delta|X| \sim R^{2} / r$ [32].

Since the supergravity solution (2.1) is obtained from the type IIB D3-brane solution, by a set of T-dualities and boosts, one expects that the resultant nonrelativistic field theory, should have gauge field and gauge symmetry. Indeed one may suspect that the obtained theory could be related to three dimensional nonrelativistic gauge theory which has previously been studied in the context of three dimensional Chern-Simons relativistic gauge theory (see for example [33]). Therefore it would be interesting to explore different features of 3D non-relativistic gauge theory by making use of the dual gravity. In particular we can evaluate the effective potential of the external quark-anti quark potential via the Wilson loop computations in the context of AdS/CFT correspondence [34,35].

To compute the effective potential following [34,35] we start from an ansatz for the classical string in the supergravity solution (2.1) which has $S c h(d-1)$ isometry,

$$
t=\tau, \quad r=\sigma, \quad x_{1}=x(\sigma), \quad \xi=\text { constant } .
$$

Indeed this ansatz in the geometry (2.1) was studied in [26] where the energy of the string as a function of distance between two external sources, $l$, was found to be

$$
E=-\frac{2 \mu R^{4}}{\pi \alpha^{\prime}} \frac{1}{l^{2}}
$$

This behavior may be understood from the fact that the dual theory in non-relativistic CFT with the dynamical scaling $z=2$.

In the rest of the paper we extend our considerations for other semi-classical strings to extract information about the possible operators of the dual non-relativistic 3D CFT.

\section{Semi-classical string in the background with Schrödinger group isometry}

In this section we will study semi-classical closed strings in the non-relativistic D3brane background. Since we are interested in the energy of the semi-classical string we need to write the non-relativistic D3-brane solution in the "global" coordinates. The corresponding solution can be found from AdS solution in the global coordinates by making use of the Null Melvin Twist [36]

$$
\begin{aligned}
d s^{2} & =\left(\frac{r}{R}\right)^{2} \frac{1}{H}\left[-\left(\frac{g}{2}+\mu^{2} r^{2} f\right) d t^{2}-\frac{g}{2} d \xi^{2}-(1+f) d t d \xi\right. \\
& \left.+\frac{R^{2}}{4} H\left(d \theta^{2}+\cos ^{2} \theta d \psi^{2}\right)\right]+\left(\frac{R}{r}\right)^{2} \frac{d r^{2}}{f}+d \mathcal{M}_{5}^{2}
\end{aligned}
$$




$$
e^{2 \phi}=H^{-1}, \quad \text { with } \quad f=1+g=1+\frac{R^{2}}{r^{2}}, \quad H=1-\frac{1}{2} \mu^{2} R^{2}
$$

There is also a non-zero RR four field as well as a NSNS two form. $d \mathcal{M}_{5}^{2}$ is the metric of internal space whose explicit form is not important for our purpose. We note, however, that the detail of the internal space will become important for other sectors of the theory. For example if we are interested in the giant magnons, the main role is played by $d \mathcal{M}_{5}^{2}$.

To proceed it is useful to make the following change of variables

$$
t \rightarrow \frac{R}{\sqrt{2}}(t+\xi), \quad \xi \rightarrow \frac{R}{\sqrt{2}}(t-\xi), \quad r \rightarrow R \sinh \rho,
$$

where upon the above metric is recast in the following form

$$
\begin{aligned}
d s^{2} & =\frac{R^{2}}{H}\left[-\left(1+\frac{\mu^{2} R^{2}}{2} \sinh ^{2} \rho\right) \cosh ^{2} \rho d t^{2}+\left(\sinh ^{2} \rho-\frac{\mu^{2} R^{2}}{8} \sinh ^{2} 2 \rho\right) d \xi^{2}\right. \\
& \left.-\frac{\mu^{2} R^{2}}{4} \sinh ^{2} 2 \rho d t d \xi+\frac{H}{4} \sinh ^{2} \rho\left(d \theta^{2}+\cos ^{2} \theta d \psi^{2}\right)\right]+R^{2} d \rho^{2}+d \mathcal{M}_{5}^{2} .(3 .
\end{aligned}
$$

\subsection{Folded closed string}

We would like to study a solution representing a rotating closed string configuration which is stretched along the radial coordinate. In order to study this system one needs to write an action for this closed string. Let us parameterize the string worldsheet by $\sigma$ and $\tau$. We can fix the re-parameterization invariance by a parameterization such that the time coordinate of space-time, $t$ to be equal to worldsheet time, i.e. $t=\tau$. In this gauge a closed string configuration representing a rotating string with angular velocity $\omega$ on geometry (3.3) stretched along the radial coordinate is given by

$$
t=\tau, \quad \psi=\omega \tau, \quad \rho(\sigma)=\rho(\sigma+2 \pi), \quad \xi=\text { constant }
$$

all other coordinates are set to zero. For this solution the Nambu-Goto action,

$$
I=-\frac{1}{2 \pi \alpha^{\prime}} \int d \sigma^{2} \sqrt{-\operatorname{det}\left(G_{\mu \nu} \partial_{a} X^{\mu} \partial_{b} X^{\nu}\right)}
$$

reads

$I=\frac{-4 R^{2}}{2 \pi \alpha^{\prime} \sqrt{H}} \int_{0}^{\rho_{0}} d \rho A^{1 / 2}(\rho), \quad A(\rho)=\left(1+\frac{\mu^{2} R^{2}}{2} \sinh ^{2} \rho\right) \cosh ^{2} \rho \dot{t}^{2}-\frac{H \dot{\psi}^{2}}{4} \sinh ^{2} \rho$,

where dot represents derivative with respect to $\tau$. The factor of 4 comes from the fact that we are dealing with a folded closed string. Working with one fold string, the string can be divided to four segments. Using the periodicity condition we just need to perform the integral for one quarter of string multiplied by factor 4 . 
To insure that the ansatz (3.4) represents a closed string we need to impose the periodicity condition which in our case is $A(\rho) \geq 0$ for all $\rho>0$. The periodicity condition, setting $\dot{t}=1, \dot{\psi}=\omega$, can be satisfied if

$$
\omega^{2} \geq 4 \frac{\sqrt{2}+\mu R}{\sqrt{2}-\mu R}=\omega_{c}^{2} .
$$

for which the $A(\rho)$ takes positive values for $\rho \leq \rho_{-}$or $\rho \geq \rho_{+}$with $\rho_{-}<\rho_{+}$, where

$$
\rho_{ \pm}=\sinh ^{-1}\left[\frac{\left(\left(\frac{H \omega^{2}}{4}-1-\frac{\mu^{2} R^{2}}{2}\right) \pm \sqrt{\left(\frac{H \omega^{2}}{4}-1-\frac{\mu^{2} R^{2}}{2}\right)^{2}-2 \mu^{2} R^{2}}\right)^{1 / 2}}{\mu R}\right] .
$$

An interesting feature of this semi-classical folded string is that the closed string cannot be longer than a maximum size given by $\rho_{\max }=\sinh ^{-1}\left[2^{1 / 4} / \sqrt{\mu R}\right]$ which corresponds to the length of string whose quantum number satisfies $\omega=\omega_{c}$. We note that for $\rho \geq \rho_{+}$, even though $A(\rho)$ is positive, we will not get a closed string. This might be thought of as the case when the periodicity condition is going to be lost and we are dealing with open string stretched all the way to infinity.

The two conserved momenta conjugate to $t$ and $\psi$ are the space-time energy $E$ and spin $S$. When the periodicity condition is satisfied, using the above Nambu-Goto action the conserved quantities are given by

$$
\begin{aligned}
E & =\frac{2 R^{2}}{\pi \alpha^{\prime} \sqrt{H}} \int_{0}^{\rho_{-}} d \rho \frac{\left(1+\frac{\mu^{2} R^{2}}{2} \sinh ^{2} \rho\right) \cosh ^{2} \rho}{\sqrt{\left(1+\frac{\mu^{2} R^{2}}{2} \sinh ^{2} \rho\right) \cosh ^{2} \rho-\frac{H \omega^{2}}{4} \sinh ^{2} \rho}} \\
S & =\frac{\omega R^{2} \sqrt{H}}{2 \pi \alpha^{\prime}} \int_{0}^{\rho_{-}} d \rho \frac{\sinh ^{2} \rho}{\sqrt{\left(1+\frac{\mu^{2} R^{2}}{2} \sinh ^{2} \rho\right) \cosh ^{2} \rho-\frac{H \omega^{2}}{4} \sinh ^{2} \rho}},
\end{aligned}
$$

From the integrals (3.9) one can proceed to compute the relation between energy and spin. To do this we can use an approximation in which the string is much shorter or of order of the critical value $\rho_{\max }$. In other words we will consider the cases where $\rho_{-} \ll \rho_{\max }$ or $\rho_{-} \sim \rho_{\max }$. Setting $\omega^{2}=\omega_{c}^{2}+\frac{4}{H} \eta$ the two limits correspond to $\eta \rightarrow \infty$ and $\eta \rightarrow 0$, respectively. Our aim will then be to find the energy $E$ as a function of spin for these two cases.

\section{Short strings}

In this case one has $\rho_{-} \sim \frac{1}{\sqrt{\eta}}$ as $\eta \rightarrow \infty$. Therefore the string is much shorter than the radius of curvature of the geometry (3.3). In fact in this limit the background space may be approximated by a flat metric near the center. Therefore the calculations reduce to spinning string in the flat space. In this limit the integrals (3.9) can be performed and find

$$
E=\frac{R^{2}}{\alpha^{\prime} \sqrt{H}} \frac{1}{\sqrt{\eta}}, \quad S=\frac{R^{2}}{4 \alpha^{\prime}} \frac{1}{\eta}
$$


so that

$$
E^{2}=\frac{4 R^{2}}{H \alpha^{\prime}} S
$$

which is the well-known flat space Regge trajectory. Indeed this is what we would expect to find; namely going deep into the core of the space time the physics should be independent of general structure and the string should locally feel the flat space. This may be compared with AdS result where we also get the Regge trajectory, though in our case we have an extra factor of $1 / H$ which should be the signature of the non-relativistic nature of the theory.

\section{Near $\rho_{\max }$ string}

As we have seen the closed string cannot be longer than a maximum size given by $\rho_{\max }=\sinh ^{-1}\left[2^{1 / 4} / \sqrt{\mu R}\right]$. So another limit we may consider is the case where string is of order of $\rho_{\max }$. In this case the spin is always large compare with the radius of the curvature of the background geometry, i.e. $S \gg \frac{R^{2}}{\alpha^{\prime}}$. For $\rho_{-} \rightarrow \rho_{\max }$ the integrals of (3.9) yield to the following expressions for $S$ and $E$ at leading order

$$
\begin{aligned}
E \approx \frac{2 R^{2}}{\pi \alpha^{\prime}} \frac{\sqrt{2}}{\mu R}\left[\frac{1+\frac{\mu R}{\sqrt{2}}}{\sqrt{1-\frac{\mu R}{\sqrt{2}}}} \tanh ^{-1}\left(\sqrt{1+\frac{\mu R}{\sqrt{2}}} \tanh \rho_{-}\right)-\frac{\mu^{2} R^{2} \sinh 2 \rho_{-}}{4 \sqrt{\left(1+\frac{\mu R}{\sqrt{2}}\right)\left(2-\mu^{2} R^{2}\right)}}\right. \\
\left.-\frac{\mu^{2} R^{2}+2 \sqrt{2} \mu R+4}{4 \sqrt{1-\frac{\mu^{2} R^{2}}{2}}} \rho_{-}\right]+\cdots \\
S \approx \frac{R^{2}}{\pi \alpha^{\prime}} \frac{\sqrt{2}}{\mu R}\left[\sqrt{1+\frac{\mu R}{\sqrt{2}}} \tanh ^{-1}\left(\sqrt{1+\frac{\mu R}{\sqrt{2}}} \tanh \rho_{-}\right)-\left(1+\frac{\mu R}{\sqrt{2}}\right) \rho_{-}\right]+\cdots .
\end{aligned}
$$

Note that both of the above expressions diverge for $\rho_{-} \rightarrow \rho_{\max }$, nevertheless for $\mu R \sim 1$ we find

$$
E \approx 2 \sqrt{\frac{\sqrt{2}+\mu R}{\sqrt{2}-\mu R}} S-\frac{R^{2}}{2 \pi \alpha^{\prime}} \frac{2 \sqrt{2}+\mu R}{\sqrt{2-\mu^{2} R^{2}}} \sinh ^{-1}(\sqrt{\sqrt{2} / \mu R})
$$

while for $\mu R \ll 1$ where we can expand the above expressions in terms of $\mu R$ one gets

$$
E \approx\left(2+\frac{7 \mu^{2} R^{2}}{64}+\mathcal{O}\left(\mu^{4} R^{4}\right)\right) S+\frac{R^{2}}{\pi \alpha^{\prime}}\left(1-\frac{\mu R}{8 \sqrt{2}}+\frac{3 \mu^{2} R^{2}}{32}+\mathcal{O}\left(\mu^{3} R^{3}\right)\right) \ln \left(\frac{\alpha^{\prime} S}{R^{2}}\right) .
$$

This has to be compared with the case of $A d S_{5}$ geometry where we have [22]

$$
E=S+\frac{R^{2}}{\pi \alpha^{\prime}} \ln \left(\frac{\alpha^{\prime}}{R^{2}} S\right)+\cdots
$$


in which the field theory dual is a relativistic conformal gauge theory and this behavior looks very similar to the logarithmic growth of anomalous dimensions of operators with spin in the gauge theory. Although in our case we still get the same expression as that in the relativistic case 4 , the coefficients are corrected by functions of $\mu R$, at least up to the order we are considering. This may be understood as follows. In fact as we argued the dual non-relativistic theory contains a gauge field inherited from the $4 \mathrm{D} \mathcal{N}=4 \mathrm{SYM}$ theory. So, the 3D theory may be studied by a small deformation of the $4 \mathrm{D}$ where the deformation parameter is $\mu R$. Therefore for $\mu R \ll 1$ we still have the same operators as those in $4 \mathrm{D}$ though the anomalous dimension of the corresponding operators get corrected due to the deformation, bringing us to the non-relativistic field theory. It would be interesting to see if such a behavior can be obtained from non-relativistic gauge theory as well.

\subsection{Circular pulsating string}

Another semi-classical string we would like to study is a circular pulsating string first studied in [24] in the AdS geometry. This is a string which wrapped around a angular coordinate and pulsates in radial direction. More precisely consider a circular pulsating closed string which is wrapped $m$ times around the $\psi$ direction. The corresponding string configuration is given by

$$
t=\tau, \quad \rho=\rho(t), \quad \psi=m \sigma, \quad \xi=\text { constant } .
$$

The other coordinates are set to zero. The Nambu-Goto action for this configuration in the geometry (3.3) reads

$$
S=-\frac{m R^{2}}{4 \alpha^{\prime} \sqrt{H}} \int d t\left(1+\frac{\mu^{2} R^{2}}{2} \sinh ^{2} \rho\right)^{1 / 2} \sinh 2 \rho \sqrt{1-\frac{H \dot{\rho}^{2}}{\left(1+\frac{\mu^{2} R^{2}}{2} \sinh ^{2} \rho\right) \cosh ^{2} \rho}}
$$

where dot represents derivative with respect to $t$. It is useful to make the following change of variable

$$
\eta=\int \sqrt{\frac{H}{\left(1+\frac{\mu^{2} R^{2}}{2} \sinh ^{2} \rho\right) \cosh ^{2} \rho}} d \rho
$$

by which the above action can be recast to the following form

$$
S=-\int d t g(\eta) \sqrt{1-\dot{\eta}^{2}}
$$

where $g(\eta)=\frac{m R^{2}}{4 \alpha^{\prime} \sqrt{H}}\left(1+\frac{\mu^{2} R^{2}}{2} \sinh ^{2} \rho\right)^{1 / 2} \sinh 2 \rho$. The associated Hamiltoninan with the above action is given by

$$
H=\sqrt{\Pi^{2}+g(\eta)^{2}}
$$

\footnotetext{
${ }^{4}$ Note that setting $\mu R=0$ in the equation (3.14) we recover the relativistic (3.15) up to numerical factors. These disagreements are due to the normalization of the $\psi$ coordinate in metric (3.3). This is also the case in the short string limit.
} 
with $\Pi$ being the canonical momentum. Note that the $H^{2}$ may be considered as a one dimensional quantum mechanical system with the potential

$$
V(\eta)=g(\eta)^{2}=\left(\frac{m R^{2}}{4 \alpha^{\prime} \sqrt{H}}\right)^{2}\left(1+\frac{\mu^{2} R^{2}}{2} \sinh ^{2} \rho\right) \sinh ^{2} 2 \rho .
$$

Therefore following [24] we can use the Bohr-Sommerfeld analysis for the quantization of the states. The quantization condition is

$$
\left(n+\frac{1}{2}\right) \pi=\int_{\eta_{1}}^{\eta_{2}} d \eta \sqrt{E^{2}-V(\eta)}
$$

where $\eta_{1,2}$ are the turning points. It is useful to return to the original coordinate $\rho$ in which the above quantization condition becomes

$$
\left(n+\frac{1}{2}\right) \pi=E \sqrt{H} \int d \rho \sqrt{\frac{1-\frac{1}{B^{2}}\left(1+\frac{\mu^{2} R^{2}}{2} \sinh ^{2} \rho\right) \sinh ^{2} 2 \rho}{\left(1+\frac{\mu^{2} R^{2}}{2} \sinh ^{2} \rho\right) \cosh ^{2} \rho}},
$$

where $B^{2}=\frac{4 \alpha^{\prime} E \sqrt{H}}{m R^{2}}$. To perform the integral we follow the procedure of $[24,37]$ decomposing the integral into two parts

$$
\begin{aligned}
\left(n+\frac{1}{2}\right) \pi=E \sqrt{H}[ & -\int_{0}^{\rho_{0}} d \rho \frac{1-\sqrt{1-\frac{1}{B^{2}}\left(1+\frac{\mu^{2} R^{2}}{2} \sinh ^{2} \rho\right) \sinh ^{2} 2 \rho}}{\sqrt{\left(1+\frac{\mu^{2} R^{2}}{2} \sinh ^{2} \rho\right) \cosh ^{2} \rho}} \\
& \left.+\int_{0}^{\rho_{0}} \frac{d \rho}{\sqrt{\left(1+\frac{\mu^{2} R^{2}}{2} \sinh ^{2} \rho\right) \cosh ^{2} \rho}}\right],
\end{aligned}
$$

where $\rho_{0}$ is the turning point in the original coordinates. For the large $B$ the first integral in (3.24) becomes

$$
\int_{0}^{\rho_{0}} d \rho \frac{1-\sqrt{1-\frac{1}{B^{2}}\left(1+\frac{\mu^{2} R^{2}}{2} \sinh ^{2} \rho\right) \sinh ^{2} 2 \rho}}{\sqrt{\left(1+\frac{\mu^{2} R^{2}}{2} \sinh ^{2} \rho\right) \cosh ^{2} \rho}} \approx \frac{2 B^{-2 / 3}}{(\sqrt{2} \mu R)^{1 / 3}}\left(-\frac{1}{2}+\frac{3^{3 / 2} \Gamma\left(\frac{2}{3}\right)^{3}}{2^{8 / 3} \pi}\right),
$$

while for the second integral one finds

$$
\int_{0}^{\rho_{0}} \frac{d \rho}{\sqrt{\left(1+\frac{\mu^{2} R^{2}}{2} \sinh ^{2} \rho\right) \cosh ^{2} \rho}} \approx \frac{2 B^{-2 / 3}}{(\sqrt{2} \mu R)^{1 / 3}}\left(\frac{2 \pi^{2}}{9 \Gamma\left(\frac{2}{3}\right)^{3}} B^{2 / 3}-\frac{1}{2}\right) .
$$

Thus altogether we get

$$
\left(n+\frac{1}{2}\right) \pi \approx \frac{4 \pi^{2}}{9 \Gamma\left(\frac{2}{3}\right)^{3}} \frac{\sqrt{H}}{(\sqrt{2} \mu R)^{1 / 3}} E-\frac{3^{3 / 2} \Gamma\left(\frac{2}{3}\right)^{3}}{8 \pi}\left(\frac{\sqrt{H}}{\sqrt{2} \mu \alpha^{\prime 2}}\right)^{1 / 3} R m^{2 / 3} E^{1 / 3},
$$


which can be inverted to find energy as a function of $n$

$$
E \approx \alpha n+\beta\left(m^{2} n\right)^{1 / 3}
$$

where $\alpha$ and $\beta$ are two constants given in terms of $\mu, R$ and $\alpha^{\prime}$ which can be read from equation (3.27), though whose explicit forms are not important for our consideration. This has to be compared with that in AdS geometry where it was found that the $E-n$ grows as $n^{1 / 2}[24]$. It would be interesting to find the dual operator in the non-relativistic gauge theory.

\section{Open string and drag force}

So far we have considered closed strings in the geometry with Schrödinger isometry. As we have already mentioned, in section two, open string can also be used to explore different features of the model using the supergravity dual. It may be used to obtain, for example, the effective potential of quark-anti quark system. In this section we would like to study a non-relativistic quark moving through a hot plasma by making use of the gravity description of the system. In other words, following [39] we would like to study the drag force for a quark in a non-relativistic field theory.

To do so, one first needs the gravity dual of the non-relativistic field theory at finite temperature. In the context of AdS/CFT duality we know that heating up the dual field theory generically corresponds to adding a black hole in the bulk geometry. Therefore we need to find the supergravity solution corresponding to the black hole in the geometry (2.1). The relevant supergravity solution for our studies is given in [30] (see also [38])

$$
\begin{aligned}
d s^{2} & =\left(\frac{r}{R}\right)^{2} \frac{1}{H}\left[-\left(\frac{g}{2}+\mu^{2} r^{2} f\right) d t^{2}-\frac{g}{2} d \xi^{2}+(1+f) d t d \xi+H d x_{i}^{2}\right] \\
& +\left(\frac{R}{r}\right)^{2}\left[\frac{d r^{2}}{f}+r^{2}\left(\frac{(d \chi+A)^{2}}{H}+d s_{P}^{2}\right)\right], \\
e^{2 \phi} & =H^{-1}
\end{aligned}
$$

where

$$
g=-\left(\frac{r_{H}}{r}\right)^{4}, \quad H=1-\frac{\mu^{2} r^{2}}{2} g, \quad f=1+g
$$

There is also a non-zero RR 4-form as well as a NSNS 2-form (see [30]).

To proceed we start from an ansatz for the open string representing an external moving source in the dual field theory. To write the open string ansatz it is useful to make the following change of variables

$$
t \rightarrow \frac{1}{\sqrt{2}}(\xi-t), \quad \xi \rightarrow \frac{1}{\sqrt{2}}(\xi+t),
$$


in which the above metric reads

$$
\begin{aligned}
d s^{2} & =\left(\frac{r}{R}\right)^{2} \frac{1}{H}\left[-\left(1+\frac{1}{2} \mu^{2} r^{2}\right) f d t^{2}+\left(1-\frac{1}{2} \mu^{2} r^{2} f\right) d \xi^{2}+\mu^{2} r^{2} f d t d \xi+H d x_{i}^{2}\right] \\
& +\left(\frac{R}{r}\right)^{2}\left[\frac{d r^{2}}{f}+r^{2}\left(\frac{(d \chi+A)^{2}}{H}+d s_{P}^{2}\right)\right]
\end{aligned}
$$

In this notation our ansatz is given by

$$
t=\tau, \quad r=\sigma, \quad x_{1}=v t+x(r), \quad \xi=\text { constant } .
$$

Re-writing the relevant part of the metric in the following form

$$
d s^{2}=g_{t t} d t^{2}+g_{x x} d x_{1}^{2}+g_{r r} d r^{2}
$$

the Nambu-Goto action becomes [40]

$$
S=-\frac{1}{2 \pi \alpha^{\prime}} \int d t d r \sqrt{-\left(g_{t t} g_{r r}+g_{t t} g_{x x} x^{2}+g_{x x} g_{r r} v^{2}\right)}
$$

where prime represents derivative with respect to $r$. Since the metric components are $t$ independent, the above action may be treated as a one dimensional mechanical system whose momentum is the constant of motion

$$
\frac{-g_{t t} g_{x x} x^{\prime}}{\sqrt{-\left(g_{t t} g_{r r}+g_{t t} g_{x x} x^{\prime 2}+g_{x x} g_{r r} v^{2}\right)}}=c=-2 \pi \alpha^{\prime} \pi_{x}=\text { constant }
$$

which can be solved for $x^{\prime}$ leading to

$$
x^{\prime 2}=4 \pi^{2} \alpha^{\prime 2} \pi_{x}^{2}\left(\frac{g_{r r}\left(-g_{t t}-g_{x x} v^{2}\right)}{g_{x x} g_{t t}\left(g_{x x} g_{t t}+4 \pi^{2} \alpha^{\prime 2} \pi_{x}^{2}\right)}\right) .
$$

In terms of the constant $\pi_{x}$ one has [40]

$$
\frac{d E}{d t}=\pi_{x} v, \quad \frac{d P}{d t}=\pi_{x} .
$$

where $E$ and $P$ are energy and momentum the open string gain from through its end point. To find $c$ we note that the equation (4.9) physically make sense if the numerator and denominator vanish at the same point [39]. Setting the numerator of (4.9) to zero, for the supergravity solution (4.4), one finds

$$
\frac{1}{2} \mu^{2} r_{0}^{6}+\left(1-v^{2}\right) r_{0}^{4}-\frac{1}{2} \mu^{2} r_{H}^{4} r_{0}^{2}\left(1+v^{2}\right)-r_{H}^{4}=0
$$

which can be solved for $r_{0}$. Plugging the solution $r_{0}$ in the denominator one arrives at

$$
\pi_{x}=-\left.\frac{v}{2 \pi \alpha^{\prime}} g_{x x}\right|_{r_{0}} .
$$


From (4.11) we see that setting $\mu=0$ the velocity changes from $v=0$ to $v=1$ as $r_{0}$ varies from $r_{H}$ to infinity, as expected for relativistic field theory. On the other hand for $\mu \neq 0$ where the dual theory is supposed to be non-relativistic we observe that as we are varying the $r_{0}$ from $r_{H}$ to infinity, the velocity takes its value from zero to infinity. This is in fact due to the non-relativistic property of the dual field theory.

To proceed we need to solve the equation (4.11) to find $r_{0}$ in terms of velocity. Then using the expression for constant conjugate momentum in terms of the metric components presented in (4.12), one may read, for example, the drag force from (4.10). To proceed we will consider two different limits depending on whether the velocity is small or large. In these limits the drag force becomes

$$
\frac{d P}{d t} \approx \begin{cases}-\frac{v}{2 \pi \alpha^{\prime}} \frac{r_{H}^{2}}{R^{2}}\left(1+\frac{1}{2} v^{2}\right) & \text { for } \quad v \ll 1 \\ -\frac{v}{2 \pi \alpha^{\prime}} \frac{2}{\mu^{2} R^{2}}\left(v^{2}+\mu^{4} r_{H}^{4}-4\right) & \text { for } \quad v \gg 1 .\end{cases}
$$

We recognize the first one as the non-relativistic limit of that found in [39] for the relativistic field theory when $v \ll 1$. The second case is just because of the non-relativistic nature of the dual field theory.

Now consider a single non-relativistic particle with momentum $P$ and mass $M$, then we have $P=M v$. It is useful to formally rewrite the above expression for the drag force in terms of $P$. Then we can perform the integral yielding

$$
P(t) \approx\left\{\begin{array}{lll}
P_{0} e^{-\frac{\pi R^{2} T^{2}}{4 \alpha^{\prime} M} t} & \text { for } & P_{0} \ll M, \\
\left(\frac{1}{P_{0}^{2}}+\frac{2 t}{\pi \alpha^{\prime} \mu^{2} R^{2} M^{3}}\right)^{-1 / 2} & \text { for } & P_{0} \gg M .
\end{array}\right.
$$

In the above expression we have set $r_{H}^{2}=\frac{\pi^{2} R^{4}}{2} T^{2}$ [30]. Similarly one finds

$$
E(t) \approx\left\{\begin{array}{lll}
E_{0} e^{-\frac{\pi R^{2} T^{2}}{2 \alpha^{\prime} M} t} & \text { for } & E_{0} \ll M / 2, \\
\left(\frac{1}{E_{0}}+\frac{4 t}{\pi \alpha^{\prime} \mu^{2} R^{2} M^{2}}\right)^{-1} & \text { for } & E_{0} \gg M / 2 .
\end{array}\right.
$$

This means that a particle with energy much less than its mass will lose its energy exponentially with time, while for a particle with kinetic energy much more than its mass, the energy is lost as $t^{-1}$. Another interesting feature of the model is that for the slowly moving particles the relaxation time, $t_{0}=\frac{2 \alpha^{\prime} M}{\pi R^{2} T^{2}}$, depends inversely on temperature, whereas for the fast moving particles it is temperature independent, $t_{0}=\pi \alpha^{\prime} \mu^{2} R^{2} M^{2} / 4$. On the other hand in the first case the relaxation time is $\mu$ independent, while in the second case it proportional to $\mu^{2}$. This shows that even at zero temperature a non-relativistic particle will lose its energy. In the next section we explore this point in more details. 


\section{$5 \quad$ Speed limit and drag force}

To study a quark moving through a hot plasma, Gubser [39] has considered a moving open string in a geometry with horizon where the radius of the horizon is related to the temperature of the dual gauge theory. Although having the horizon is important to deal with the gauge theory at finite temperature, as far as the gravity computations are concerned we are free to redo the computations for a geometry without horizon. Essentially what one needs to do is Wilosn loop computations in the context of AdS/CFT correspondence where the string is moving as well.

Let us first consider a moving open string given by (4.5) in the $A d S_{5} \times S^{5}$ geometry parametrized as follows

$$
d s^{2}=\left(\frac{r}{R}\right)^{2}\left(-d t^{2}+d x_{1}^{2}+d x_{2}^{2}+d x_{3}^{2}\right)+\left(\frac{R}{r}\right)^{2} d r^{2}+R^{2} d \Omega_{5}^{2} .
$$

Using the procedure of the previous section one gets

$$
x^{\prime 2}=\left(2 \pi \alpha^{\prime}\right)^{2} \pi_{x}^{2} \frac{1-v^{2}}{\left(\frac{r}{R}\right)^{4}\left(\left(\frac{r}{R}\right)^{4}-\left(2 \pi \alpha^{\prime}\right)^{2} \pi_{x}^{2}\right)},
$$

which is well behaved if $v<1$ representing the fact that the dual theory is relativistic and therefore there is a bound for the velocity. Moreover we observe that the constant conjugate momentum, $\pi_{x}$, is independent of $v$.

Now consider the following ansatz for the open string moving in the background (2.1)

$$
t=\tau, \quad r=\sigma, \quad x_{1}=v t+x(r), \quad \xi=\text { constant } .
$$

In this case one finds

$$
x^{\prime 2}=\left(2 \pi \alpha^{\prime}\right)^{2} \pi_{x}^{2} \frac{\mu^{2} r^{2}-v^{2}}{\left(\frac{r}{R}\right)^{4} \mu^{2} r^{2}\left(\left(\frac{r}{R}\right)^{4} \mu^{2} r^{2}-\left(2 \pi \alpha^{\prime}\right)^{2} \pi_{x}^{2}\right)} .
$$

We observe that in this case there is no bound on the velocity and it can change from zero to infinity. This is indeed the reflection of the fact that dual theory is non-relativistic. To avoid the imaginary solution one arrives at

$$
\pi_{x}=-\frac{v}{2 \pi \alpha^{\prime}} \frac{v^{2}}{\mu^{2} R^{2}} .
$$

For a particle with mass $M$ and momentum $P=M v$ the drag force reads

$$
\frac{d P}{d t}=-\frac{1}{2 \pi \alpha^{\prime} M^{3} \mu^{2} R^{2}} P^{3}
$$

which yields to

$$
P=\left(\frac{1}{P_{0}^{2}}+\frac{t}{\pi \alpha^{\prime} M^{3} \mu^{2} R^{2}}\right)^{-\frac{1}{2}}
$$


This means that in this case even though the system is at zero temperature the moving particle losses its energy and the relaxation time is given in terms of $\mu^{2}$. Whereas in the hot plasma it is controlled by temperature. This may be be related to the fact that $\mu$ in the dual non-relativistic field theory could be interpreted as the chemical potential.

The drag force calculations can be generalized to other backgrounds obtained from Null Melvin Twist procedure of $D p$-brane for $p \leq 4$. The relevant solutions are given by [30]

$$
\begin{aligned}
d s^{2} & =\left(\frac{r}{R}\right)^{\frac{7-p}{2}} \frac{1}{H}\left[-\left(1+\frac{1}{2} \mu^{2} r^{2}\right) f d t^{2}+\left(1-\frac{1}{2} \mu^{2} r^{2} f\right) d \xi^{2}+\mu^{2} r^{2} f d t d \xi+H d x_{i}^{2}\right] \\
& +\left(\frac{R}{r}\right)^{\frac{p+1}{2}}\left[\frac{d r^{2}}{f}+r^{2}\left(\frac{(d \chi+A)^{2}}{H}+d s_{P}^{2}\right)\right]
\end{aligned}
$$

where $f=1-\left(r_{H} / r\right)^{7-p}$ and $H=1+\mu^{2} r_{H}^{7-p} / 2 r^{5-p}$. Going through the computations of section four, for slowly moving particles, we find

$$
P=P_{0} e^{-\frac{t}{t_{0}}}, \quad \text { with } \quad t_{0}=\left(\frac{(7-p)^{4}}{2^{p+1} \pi^{p-1}}\right)^{\frac{1}{5-p}} \frac{\alpha^{\prime} M}{(R T)^{\frac{4}{5-p}}}
$$

while for fast moving particles we get a universal result given by that in equation (4.14). Therefore all the models parametrized by $p \leq 4$ exhibit the same nonrelativistic behavior, though in the case of slowly moving particles the characteristic nature of energy lost is fixed by different power of the temperature. i.e. $t_{0} \sim$ $(R T)^{\frac{4}{p-5}}$.

\section{Discussions and conclusions}

In this paper we have studied a number of features of non-relativistic CFT by making use of the supergravity solution in type IIB string theory. Although we have mainly considered 3 dimensional CFT whose gravity dual can be obtained from D3-brane using TsT duality, we would expect that the general features we explored in this paper can be applied for other dimensions too.

Since the world volume theory of D3 brane is a supersymmetric gauge theory, and taking into account that supergravity solution (2.1) is obtained from D3-brane by the Null Melvin Twist procedure, we would expect that the resultant theory still contains a gauge field. Of course the procedure will reduce the number of supersymmetries as well as the space time symmetry. Indeed for the case where the internal space is a sphere the amount of supercharge preserved by the background are eight. The space time symmetry will also reduce to Schrödinger symmetry. Therefore we expect that the field theory dual to the type IIB on supergravity solution (2.1) to be nonrelativistic super conformal gauge theory in three dimensions. 
Since the supergravity dual can be embedded in type IIB string theory it is natural to study semi-classical string in this background to explore some properties of the dual non-relativistic superconformal gauge theory. In particular we have seen that the effective potential of the external objects is proportional to $l^{-2}$ as expected for a non-relativistic CFT. One may find the effective potential of quark-anti quark as a function of distance $l$ for arbitrary $p$ where the corresponding supergravity solutions are given by (5.8) with $r_{H}=0$. This has been done in [26] where the authors argued that the interaction between external objects is due to their lightlike dipole moments given by $\mu$

$$
E \sim-\frac{\mu}{\alpha^{\prime}}\left(\frac{R^{4}}{l^{2}}\right)^{\frac{2}{5-p}} .
$$

For $p=3$ which we have mainly considered in this paper, we have interpreted the effective potential due to non-relativistic CFT given by dynamical scaling $t \rightarrow \lambda^{2} t$ and $x \rightarrow \lambda x$. For other cases there is no such a clear interpretation.

We have also considered folded rotating closed strings in the geometry with isometry of Schrödinger group where we have shown that the anomalous dimension of the corresponding dual operator exhibits logarithmic corrections similar to that in the relativistic gauge theory for $\mu R \ll 1$. It would be interesting to find such a behavior directly from non-relativistic gauge theory.

We have also studied circular pulsating closed strings where we have observed that although in the leading order the anomalous dimension is proportional to the winding number of the string, $\Delta \sim n$, at subleading order it goes as $n^{1 / 3}$. It is worth noting that in the case of four dimensional relativistic gauge theory the subleading correction grows as $\Delta-n \sim n^{1 / 2}$. This should be taken as the effect of non-relativistic nature of the theory.

It is worth noting that whenever we have deviations from the relativistic field theory or in the gravity side from $A d S_{5}$ gravity, the deviations are controlled by the dimensionless parameter $\mu R$. Therefore assuming $\mu R \ll 1$ this parameter can be thought of as the expansion parameter by which we can study the non-relativistic three dimensional conformal gauge theory as a perturbation of four dimensional $\mathcal{N}=4$ superconformal gauge theory. In particular since the non-relativistic CFT we have been considering may be obtained by reduction (contract) from $\mathcal{N}=44 \mathrm{D}$ theory, we might suspect that the AdS/CFT dictionary, in some extend, works the same as before. If correct, the operator dual to dilaton would be $\operatorname{Tr} F^{2}$, though in this case the anomalous dimension of the operator gets higher loop corrections, such that summing up all the loops we get $\Delta=2+\sqrt{2+n^{2} \mu^{2} R^{2}}$. Actually this is the expression we have given in section two for the case of $M=\frac{n}{R}$ where from gravity point of view $M$ is the momentum of the dilaton in the light like direction $\xi$. By $F^{2}$ we mean the reduction of four dimensional $F^{2}$ to three dimensions.

On the other hand since the three dimensional theory is a supersymmetric theory we expect to have scalars in the model which might be identified with the coordinates in which the isometry of internal space acts on. Therefore following the general 
philosophy of [22] one might expect that the operators dual to the folded rotating closed string with spin $S$ have the following schematic form

$$
\mathcal{O}_{S} \sim \operatorname{Tr} X \nabla^{S} X
$$

where $X$ is the three dimensional scalar filed. At leading order the anomalous dimension should be $\Delta \sim S$. But as we have seen the anomalous dimension of the operator gets corrections and the corrections depend on $\mu R$ which controls the non-relativistic effects. It would be interesting to study these operators from nonrelativistic gauge theory point of view.

We have also considered non-relativistic three dimensional CFT at finite temperature. In particular we have considered an open string moving in the background created by a black hole geometry in (2.1). In the dual picture this means that we are dealing with a quark moving through the hot plasma. Following [39] we have evaluated the drag force for this case too. The first observation we have made is that there is no speed limit in this case, pointing toward the fact that the theory is non-relativistic5. Moreover depending on the initial energy of the moving quark, it loses the energy either exponentially $e^{-t / t_{0}}$ or as $\left(t_{0} / t\right)$. We have also observed that the relaxation time for slowly moving particles depends on the temperature and is independent of $\mu$, though for fast moving particles it only depends on $\mu$.

The drag force like computations may also be done for the non-relativistic field theory even at zero temperature. Doing so, one finds that unlike the AdS case where we only get a casual speed limit, in the non-relativistic case one arrives at non-trivial results, though in comparison with finite temperature system, the physics is controlled by $\mu$. It would be interesting to understand this observation from nonrelativistic gauge theory dual.

\section{Acknowledgments}

We would like to thank Farhad Ardalan for useful discussions and comments. We would also like to thanks Shahin Rouhani for discussions on the AdS/non-relativistic CFT duality. This work is supported in part by Iranian TWAS chapter at ISMO.

\section{References}

[1] J. M. Maldacena, "The large N limit of superconformal field theories and supergravity," Adv. Theor. Math. Phys. 2, 231 (1998) [Int. J. Theor. Phys. 38, 1113 (1999)] arXiv:hep-th/9711200].

[2] Y. Nishida and D. T. Son, "Nonrelativistic conformal field theories," arXiv:0706.3746.

\footnotetext{
${ }^{5}$ The main motivation of the paper [26] where the metric (2.1) was first presented was to study the non-local features of the dual theory. In light of the recent studies as well as our observation in this paper we see that the dual theory is indeed non-local due to is non-relativistic nature.
} 
[3] D. T. Son, "Toward an AdS/cold atoms correspondence: a geometric realization of the Schrodinger symmetry," Phys. Rev. D 78, 046003 (2008) arXiv:0804.3972 [hep-th]].

[4] K. Balasubramanian and J. McGreevy, "Gravity duals for non-relativistic CFTs," Phys. Rev. Lett. 101, 061601 (2008) arXiv:0804.4053 [hep-th]].

[5] W. D. Goldberger, "AdS/CFT duality for non-relativistic field theory," arXiv:0806.2867 [hep-th].

[6] W. Y. Wen, "AdS/NRCFT for the (super) Calogero model," arXiv:0807.0633 [hep-th].

[7] Y. Nakayama, "Index for Non-relativistic Superconformal Field Theories," JHEP 0810, 083 (2008) arXiv:0807.3344 [hep-th]].

[8] J. W. Chen and W. Y. Wen, "Shear Viscosity of a Non-Relativistic Conformal Gas in Two Dimensions," arXiv:0808.0399 [hep-th].

[9] D. Minic and M. Pleimling, "Non-relativistic AdS/CFT and Aging/Gravity Duality," arXiv:0807.3665 [cond-mat.stat-mech].

[10] E. Imeroni, "On deformed gauge theories and their string/M-theory duals," JHEP 0810, 026 (2008) [arXiv:0808.1271 [hep-th]].

[11] A. V. Galajinsky, "Remark on quantum mechanics with conformal Galilean symmetry," Phys. Rev. D 78, 087701 (2008) arXiv:0808.1553 [hep-th]].

[12] S. Kachru, X. Liu and M. Mulligan, "Gravity Duals of Lifshitz-like Fixed Points," Phys. Rev. D 78, 106005 (2008) arXiv:0808.1725 [hep-th]].

[13] S. S. Pal, "Null Melvin Twist to Sakai-Sugimoto model," arXiv:0808.3042 [hepth].

[14] S. Sekhar Pal, "Towards Gravity solutions of AdS/CMT," arXiv:0808.3232 [hep-th].

[15] S. Pal, "More gravity solutions of AdS/CMT," arXiv:0809.1756 [hep-th].

[16] C. Duval, M. Hassaine and P. A. Horvathy, "The geometry of Schródinger symmetry in gravity background/non-relativistic CFT," arXiv:0809.3128 [hepth].

[17] F. L. Lin and S. Y. Wu, "Non-relativistic Holography and Singular Black Hole," arXiv:0810.0227 [hep-th].

[18] S. A. Hartnoll and K. Yoshida, "Families of IIB duals for nonrelativistic CFTs," arXiv:0810.0298 [hep-th]. 
[19] M. Schvellinger, "Kerr-AdS black holes and non-relativistic conformal QM theories in diverse dimensions," arXiv:0810.3011 [hep-th].

[20] M. Ramgamani, S. F. Ross, D. T. Son, E. G. Thompson, "Conformal nonrelativistic hydrodynamics from gravity," arXiv:0811.2049 [hep-th].

[21] C. Leiva and M. S. Plyushchay, "Conformal symmetry of relativistic and nonrelativistic systems and AdS/CFT correspondence," Annals Phys. 307, 372 (2003) arXiv:hep-th/0301244.

[22] S. S. Gubser, I. R. Klebanov and A. M. Polyakov, "A semi-classical limit of the gauge/string correspondence," Nucl. Phys. B 636, 99 (2002) arXiv:hep-th/0204051.

[23] S. Frolov and A. A. Tseytlin, "Semiclassical quantization of rotating superstring in $\operatorname{AdS}(5)$ x S(5)," JHEP 0206, 007 (2002) arXiv:hep-th/0204226].

[24] J. A. Minahan, "Circular semiclassical string solutions on $A d S_{5} \times S^{5}$," Nucl. Phys. B 648, 203 (2003) arXiv:hep-th/0209047.

[25] E. G. Gimon, A. Hashimoto, V. E. Hubeny, O. Lunin and M. Rangamani, "Black strings in asymptotically plane wave geometries," JHEP 0308, 035 (2003) arXiv:hep-th/0306131.

[26] M. Alishahiha and O. J. Ganor, "Twisted backgrounds, pp-waves and nonlocal field theories," JHEP 0303 (2003) 006 [arXiv:hep-th/0301080.

[27] C. P. Herzog, M. Rangamani and S. F. Ross, "Heating up Galilean holography," arXiv:0807.1099 [hep-th].

[28] A. Adams, K. Balasubramanian and J. McGreevy, "Hot Spacetimes for Cold Atoms," arXiv:0807.1111 [hep-th].

[29] J. Maldacena, D. Martelli and Y. Tachikawa, "Comments on string theory backgrounds with non-relativistic conformal symmetry," JHEP 0810, 072 (2008) arXiv:0807.1100 [hep-th]].

[30] L. Mazzucato, Y. Oz and S. Theisen, "Non-relativistic Branes," arXiv:0810.3673 [hep-th].

[31] J. L. B. Barbon and C. A. Fuertes, "On the spectrum of nonrelativistic AdS/CFT," JHEP 0809, 030 (2008) [arXiv:0806.3244 [hep-th]].

[32] L. Susskind and E. Witten, "The holographic bound in anti-de Sitter space," arXiv:hep-th/9805114.

[33] C. R. Hagen, "Galilean-invariant gauge theory," Phys. Rev. D 30, 848 (1985). 
[34] S. J. Rey and J. T. Yee, "Macroscopic strings as heavy quarks in large N gauge theory and anti-de Sitter supergravity," Eur. Phys. J. C 22, 379 (2001) arXiv:hep-th/9803001.

[35] J. M. Maldacena, "Wilson loops in large N field theories," Phys. Rev. Lett. 80, 4859 (1998) arXiv:hep-th/9803002.

[36] D. Yamada, "Thermodynamics of Black Holes in Schrodinger Space," arXiv:0809.4928 [hep-th].

[37] M. Alishahiha and A. E. Mosaffa, "Circular semiclassical string solutions on confining AdS/CFT backgrounds," JHEP 0210, 060 (2002) arXiv:hep-th/0210122.

[38] P. Kovtun and D. Nickel, "Black holes and non-relativistic quantum systems," arXiv:0809.2020 [hep-th].

[39] S. S. Gubser, "Drag force in AdS/CFT," Phys. Rev. D 74, 126005 (2006) arXiv:hep-th/0605182.

[40] C. P. Herzog, "Energy loss of heavy quarks from asymptotically AdS geometries," JHEP 0609, 032 (2006) arXiv:hep-th/0605191]. 